Journal of Social and Development Sciences

Vol. 4, No. 2, pp. 69-73, Feb 2013 (ISSN 2221-1152)

\title{
A Bootstrap Approach to Evaluating the Power of the Houck's Test for Asymmetry
}

\author{
Henry de-Graft Acquah \\ University of Cape Coast, Ghana \\ henrydegraftacquah@yahoo.com
}

\begin{abstract}
The power of the conventional Houck's model of asymmetry is examined via parametric bootstrap simulation. The results of the bootstrap simulations indicate that the Houck's model has low power in rejecting the null of symmetric adjustment. The power of the test depends on the bootstrap sample size, level of asymmetry and the amount of noise in the data generating process used in an application. With a small bootstrap sample and large noise level, the Houck's model display low power in rejecting the null hypothesis of symmetry.
\end{abstract}

Key words: Parametric Bootstrap Simulation; Asymmetry; Houck's Model; Power Test

\section{Introduction}

Houck (1977) proposes a methodology to investigate asymmetric adjustment in economic relationship using conventional statistical testing procedure. This involves specifying asymmetries to affect the direct impact of price increases and decreases. However, some studies, for example Capps and Sherwell (2007) have found it difficult to reject the null hypothesis of symmetry using Houck statistical testing procedure. The failure of the Houck's methodology to capture asymmetric behaviour in practice motivates the investigation of its power in the present study. Acquah (2010) sheds light on power of the Houck's model in rejecting the null hypothesis of symmetric adjustments in a Monte Carlo Experimentation but did not consider the use of a more robust technique such as bootstrapping to analyse the power of the Houck's model in rejecting the null hypothesis of symmetric adjustments. Bootstrap methods offer an alternative framework to the Monte Carlo methods for investigating the power of the Houck's test for asymmetry. The bootstrap technique involves deriving new samples from the original data and this gives an advantage over the Monte Carlo methods which make implicit assumptions about the true values of the model parameters. Regardless of the robustness of bootstrap methods and its advantages over the Monte Carlo methods, little work has been done to investigate the power of the Houck's model in rejecting the null hypothesis of symmetric adjustments using bootstrap technique. A fundamental issue is that the performance of the Houck's model in rejecting the null hypothesis of symmetric adjustments in bootstrap samples is not well understood. As bootstrap sample size increases will the Houck's model have increased power as noted with previous Monte Carlo Experimentation? Empirically, this study test Acquah (2010) assertion that the inability of the Houck's model to detect asymmetry in practice is due to low power. However, the current study departs from Acquah's Monte Carlo approach and adopts a bootstrap methodology. The purpose of this paper is therefore to investigate by use of bootstrap methods, the possibility that failure to reject the null hypothesis of symmetry in the Houck's model is due to low power of the conventional tests. The paper is organized as follows. In section 2, a review of related literature is presented. This is followed by a description of the Houck's model for testing for asymmetry and the Bootstrap Methods in section 3. Section 4 discusses the results of the bootstrap analysis of the Houck's model and section 5 concludes the study.

\section{Literature Review}

Asymmetric price transmission refers to the unreciprocal relationship between rises and falls in prices. For example farm and retail price. Alternative models of asymmetric price transmission have been developed and applied in a wide variety of agricultural markets by numerous authors. For instance, in a study of the Iranian meat market, Norouzi and Moghaddasi (2012) using the Houck's model finds asymmetric price transmission in all different levels of the meat market. Similarly, in a study of the German Broiler market, Appel (1992) finds that both speed and degree of price transmission from the producer to the retail level is asymmetric. In 
a study of the US pork market, Boyd and Brorsen (1998) find no evidence of asymmetric price transmission. However, Hahn (1990) challenges this result and finds that prices at all levels of the US pork and beef marketing chains are more sensitive to price increasing shocks than to price decreasing shocks. Hansmire and Willett's (1992) noted that farm-retail price transmission for New York state apples is asymmetric and Kinnucan and Forker (1987) came to the same conclusion regarding dairy product transmission in the United States. Pick et al. (1990) finds evidence that short-run but not long-run vertical price transmission on the US citrus market is asymmetric. Finally, Ward (1982) suggests both short and long run asymmetries in vertical price transmission for fresh vegetable in the United States, while Zhang et al. (1995) indicates that price transmission for peanut to peanut butter prices in the US is asymmetric in the short-run, but symmetric in the long-run. Fundamentally, each of these studies implements some variant of an econometric technique for estimating irreversibility that was introduced by Wolfram (1971) in response to work on irreversible supply reaction by Tweenten and Quance (1969). In investigating the relationship between an output price $P_{A}$ and input price $P_{B}$, Tweenten and Quance (1969) used an indicator variable to split the input price into two parts: one variable includes only increasing input prices and another includes only decreasing input prices. Symmetric price transmission is rejected if the increasing input prices are significantly different from decreasing input prices. Based on Tweenten and Quance (1969), Wolffram (1971) proposes a variable splitting technique that explicitly includes first difference of prices in the equation to be estimated which was later modified by Houck (1977).

Numerous studies estimate a dynamic variant of the Houck's static model. Some analysts distinguish between short-run and long-asymmetries by introducing lagged terms in their modeling. Long-run symmetry is tested by determining whether the sums of the coefficients in these polynomials are identical. Ward (1982) extended the Houck's specification by including lags. While, Boyd and Brorsen (1998) were the first to use lags to differentiate between magnitude and speed of transmission. Hahn (1990) attempts to generalize the methods discussed so far, referring to them as the pre-cointegration methods. Other authors (e.g. Mohanty, Peterson and Kruse, 1995) estimate the Houcks model in sum differences. Capps and Sherwell (2007) notes that the Houck's model fails to detect asymmetry when the price series are indeed asymmetric. Acquah (2010) in a Monte Carlo experimentation suggests that the inability of the Houck's model to detect asymmetry when it is present is due to low power. However, Acquah (2010) fails to adopt a robust technique such as bootstrapping in investigating the power of the Houck's model. The bootstrap approach has been employed to investigate other models of price asymmetry. For example, Acquah (2012) investigated the power of the Granger and Lee approach in rejecting the null hypothesis of symmetric adjustments in bootstrap samples. The results of the bootstrap simulations indicate that rejection frequencies increase with increases in bootstrap sample size, increases in the difference between the asymmetric adjustment speeds and decreases in the amount of noise in the true data generating process used in the application.

\section{Methodology}

Testing for Symmetry in the Houck's Model: The conventional Houck's model commonly used to model asymmetric price transmission can be specified as:

$$
\Delta \mathrm{y}_{t}=\beta_{1}^{+} \Delta x_{t}^{+}+\beta_{1}^{-} \Delta x_{t}^{-}+\varepsilon \quad \varepsilon \square N\left(0, \sigma_{\varepsilon}^{2}\right)
$$

Where $\Delta x_{t}^{+}$and $\Delta x_{t}^{-}$are the positive and negative changes in $x_{t}$. The explanatory variable $x_{t}$ is generated as independent draws from normal distribution with a constant mean and a variance of one. Asymmetry is introduced by permitting differing speeds of adjustments for the coefficients of $\Delta x_{t}^{+}$and $\Delta x_{t}^{-}$in equation (1) and $\varepsilon$ is generated as i.i.d. draws from the standard normal distribution with a sample size $\mathrm{n} . \Delta \mathrm{y}_{t}$ can be constructed using the values for beta, positive and negative changes in $x_{t}$ (i.e. $\Delta x_{t}^{+}$and $\Delta x_{t}^{-}$) and the error term as specified in equation 1 . Symmetric price transmission is tested by determining whether the coefficients ( $\beta_{1}^{+}$and $\beta_{1}^{-}$) are identical (i.e. $H_{0}: \beta_{1}^{+}=\beta_{1}^{-}$). 
The Bootstrap: Bootstrapping is the technique of estimating properties of an estimator by measuring those properties when sampling from an approximating distribution. The empirical distribution of the observed data is a standard choice for an approximating distribution. In the case where a set of observations can be assumed to be from an independent and identically distributed population, this can be implemented by constructing a number of resamples of the observed dataset, each of which is obtained by random sampling with replacement from the original data. Efron and Tibshirani (1993) notes that the basic idea of bootstrapping involves repeated random sampling with replacement from the original data, to produce random samples of the same size of the original sample referred to as a bootstrap sample. It may also be applied for constructing hypothesis tests.

Parametric Bootstrap: Parametric bootstrapping refers to the process of resampling from the residuals of a parametric regression model. First the regression model is fitted and the residuals are obtained. Using a single bootstrap iteration, a bootstrap sample of the residual is obtained. These residuals are then added to the original $x$ values to generate new bootstrap values for the outcome variable. Ordinary least squares are then applied to estimate the new bootstrap regression coefficients, for this bootstrap sample. This process of resampling of the residuals, adding them to the fitted values and estimating the regression coefficients is repeated many times to estimate the parameters of interest from the bootstrap samples.

\section{Results and Discussion}

In order to investigate the power of the test for asymmetry under various conditions, a series of bootstrap comparison of the Houck's models is carried out based on 10000 bootstrap replications. Specifically, the power of the Houck's model is evaluated under conditions of different bootstrap sample sizes, noise levels and two levels of asymmetry given by $\left(\beta_{2}{ }^{+}, \beta_{2}{ }^{-}\right) \in(0.50,0.25) \operatorname{or}(0.75,0.25)$. Fundamentally, subtle and strong levels of asymmetry are considered in the data generating process. The Houck model is evaluated in terms of its ability to reject the (false) null of symmetric adjustment against the (true) alternative of asymmetric adjustment using an F-test of the restricted versus the unrestricted model.

Table 1: Rejection frequencies based on 10000 Bootstrap Replications

\begin{tabular}{llllll}
\hline Sample Size & $\left(\beta_{1}^{+}, \beta_{1}^{-}\right)$ & Error Size $(\sigma)$ & $\bar{F}$ & Rej (5\%) & Rej (1\%) \\
\hline 50 & $(0.50,0.25)$ & 3 & 1.9313 & 0.1441 & 0.0562 \\
50 & $(0.50,0.25)$ & 2 & 2.1657 & 0.1708 & 0.0695 \\
50 & $(0.50,0.25)$ & 1 & 3.4470 & 0.2938 & 0.1475 \\
& & & & \\
150 & $(0.50,0.25)$ & 3 & 2.2035 & 0.1867 & 0.0761 \\
150 & $(0.50,0.25)$ & 2 & 2.9055 & 0.2552 & 0.1211 \\
150 & $(0.50,0.25)$ & 1 & 6.4653 & 0.5637 & 0.3697 \\
& & & & \\
500 & $(0.50,0.25)$ & 3 & 2.5107 & 0.2179 & 0.0975 \\
500 & $(0.50,0.25)$ & 2 & 5.5209 & 0.5052 & 0.3167 \\
500 & $(0.50,0.25)$ & 1 & 17.3308 & 0.9344 & 0.8487 \\
50 & $(0.75,0.25)$ & 3 & & & \\
50 & $(0.75,0.25)$ & 2 & 2.4742 & 0.1974 & 0.0891 \\
50 & $(0.75,0.25)$ & 1 & 3.3917 & 0.2829 & 0.1434 \\
150 & $(0.75,0.25)$ & 3 & 8.4105 & 0.6480 & 0.4570 \\
150 & $(0.75,0.25)$ & 2 & & & \\
150 & $(0.75,0.25)$ & 1 & 2.7065 & 0.2421 & 0.1124 \\
& & & 6.4661 & 0.5599 & 0.3672 \\
& & & 20.9233 & 0.9626 & 0.8998
\end{tabular}


The bootstrap simulation indicates the low power of the conventional F-test in rejecting the null of symmetric adjustment in small bootstrap sample sizes. There is some improvement in power when the amount of noise in the data generating process (DGP) is decreased systematically. Similarly, when the difference in asymmetric adjustment parameters is increased from 0.25 to 0.50 in the true model, an increase in power is also observed in Houck's model as illustrated in Table 1. Noticeably, it is only when the bootstrap sample size is increased to 500 that a reasonable result is obtained. These findings are consistent with Acquah (2010) Monte Carlo Experimentation which suggests that the power of the Houck's model depends on sample size, error variance and the difference in asymmetric adjustment speeds. In conclusion, the bootstrap sample sizes, difference between the asymmetric adjustment parameters and the amount of noise in the data generating process are influential in the power of the test for asymmetry. With small bootstrap sample size or large noise, the Houck's model display low power in rejecting the (false) null hypothesis of symmetry. Similarly, Acquah (2012) bootstrap simulation results indicate that the asymmetric Granger and Lee model has low power in rejecting the null hypothesis of symmetric adjustments. Furthermore, the power of the test depends on the bootstrap sample size, difference in adjustment speeds and the amount of noise in the data generating process used in the application.

\section{Conclusion}

This study investigated the power of the conventional Houck's model of asymmetry using parametric bootstrap simulation. Specifically, the power of the Houck's model in rejecting the null hypothesis of symmetric adjustments is examined under various conditions. The results of bootstrap simulations suggest that rejection frequencies increase with increases in bootstrap sample size, increases in the difference between the asymmetric adjustment speeds and decreases in the amount of noise in the true data generating process used in the application. The low power of the Houck's model in rejecting the null of symmetric adjustment in the bootstrap samples provides a justification for the failure of the Houck's model to capture asymmetric behaviour in empirical application. Future research will investigate the power of Houck's asymmetric price transmission model using non-parametric bootstrap procedure.

\section{References}

Acquah, H. D. (2012). A Bootstrap Approach to Testing for Symmetry in the Granger and Lee Asymmetric Error Correction Model. Russian Journal of Agricultural and Socio-Economic Sciences, 11(11), 33-36.

Acquah, H. D. (2010). Testing for Symmetry in the Houck's Model in Indian Development Review. An International Journal of Development Economics, 8(1), 105-107.

Appel, V. (1992). Asymmetrie in der Preistransmission. Agrarwirtschaft Sonderheft, 135, 178-213.

Boyd, M. S. \& Brorsen, B. W. (1998). Price Asymmetry in the US Pork Marketing Channel, North Central. Journal of Agricultural Economics, 10, 103- 109.

Capps, O. \& Sherwell, P. (2007). Alternative approaches in detecting asymmetry in farm-retail prices transmission of fluid milk. Journal of Agribusiness, 23(3), 313-331.

Efron, B. \& Tibshirani, R. (1993). An Introduction to the Bootstrap, London: Chapman and Hall.

Hahn, W. F. (1990). Price Transmission Asymmetry in Pork and Beef Markets. The Journal of Agricultural Economics Research, 42(4), 21-30.

Hansmire, M. R. \& Schertz-Willet, L. (1992). Price Transmission Processes: A Study of Price Lags and asymmetric Price Response Behaviour for New York Red Delicious and McIntosh Apples, Cornell University.

Houck, J. P. (1977). An Approach to Specifying and Estimating Nonreversible Functions. American Journal of Agricultural Economics, 59, 570-572

Kinnucan, H. W. \& Forker, O. D. (1987). Asymmetry in Farm-Retail Price Transmission for major Dairy Products. American Journal of Agricultural Economics, 69, 285-292. 
Mohanty, S., Peterson, E. W. F. \& Kruse, N. C. (1995). Price Asymmetry in the International Wheat Market. Canadian Journal of Agricultural Economics, 43, 355-366.

Norouzi, G. \& Moghaddasi, R. (2012). Analysis of Price Transmission along the Iranian Market Chain for Different Meat Products. World Applied Sciences Journal, 2(1), 64-69.

Pick, D. H., Karrenbrock, J. D. \& Carman, H. F. (1990). Price Asymmetry and Marketing Margin Behavior: an Example for California - Arizona Citrus. Journal of Agribusiness, 6(1), 75-84.

Tweenten, L. G. \& Quance, C. L. (1969). Positivistic Measures of aggregate Supply Elasticities: Some new Approaches. American Journal of Agricultural Economics, 51, 342-352.

Ward, R. W. (1982). Asymmetry in Retail, Wholesale and Shipping Point Pricing for fresh Vegetables. American Journal of Agricultural Economics, 62, 205-212.

Wolffram, R. (1971). Positivistic Measures of Aggregate Supply Elasticities-Some New Approaches -Some Critical Notes. American journal of Agricultural Economics, 53, 356-356.

Zhang, P., Fletcher, S. \& Carley, D. (1995). Peanut Price Transmission Asymmetry in Peanut Butter. Journal of Agribusiness, 11(1), 13-20. 